\title{
Effects of a Forward Displaced Position on Driver Injury Levels in Frontal Crashes
}

\author{
Xiao Luo ${ }^{a}$, Wenjing Du ${ }^{b}$, Shucai $\mathrm{Xu}^{\mathrm{c}^{*}, \text { Jie Yang }}{ }^{\mathrm{d}}$, Jinhuan Zhang ${ }^{\mathrm{e}}$ \\ State Key Laboratory of Automotive Safety and Energy, Department of Automotive Engineering, \\ Tsinghua University, Beijing 100084, China \\ cxushc@tsinghua.edu.cn, ${ }^{\mathrm{e}} \mathrm{zhjh} @$ tsinghua.edu.cn
}

\begin{abstract}
Keywords: Automotive Safety, Occupant Protection, Front-end Collision, Forward Displaced Position.

Abstract. To verify that a forward displaced position has effects on the driver injury levels in front-end collisions and estimate the injury levels in real accidents under this situation, a series of sled tests were conducted with dummy in different forward displaced positions. Injury response values of different body regions were calculated and used to estimate the distribution of injury levels in real accidents. The results of sled tests showed that injuries response values of the dummy driver increased obviously as its body leaned forward. And the estimated distribution of injury levels had a good similarity with statistics results. This method could also be employed to have a more comprehensive estimation of automotive active safety on occupant protection.
\end{abstract}

\section{Introduction}

The total number of accidents caused by frontal collisions and rear-end collisions has always remained high among all kinds of traffic accidents. These two kinds of accidents are also the top two reasons for the casualties in traffic accidents [1]. For both vehicles in a frontal crash and the striking vehicle in a rear-end crash, the vehicle frontal crashworthiness is very important. The frontal crashworthiness has been improved a lot with an improved structural crashworthiness design and a better match with the occupant restraint system. However, severe injuries or even deaths still exist due to a high impact speed or a wrong wearing of seat belt. Under these circumstances, vehicle active safety technologies are born. These active safety devices are able to judge the risk of a potential accident according to the detection of the vehicle environment and then send warning signals to drivers if necessary. If drivers fail to take avoidance maneuvers as expected, the system will assist in braking or even directly take over the vehicle control in an emergency [2-5]. This technology is one of the main directions for the future development of vehicle safety technologies.

Nowadays, vehicle safety technologies basically rely on braking to reduce crash severity or prevent accidents. According to the time interval from the activation of the actuator to the impact, the actuators mainly include three systems. Forward collision warning system reminds drivers to take avoidance maneuvers. Brake assist system offers extra braking forces. Autonomous emergency braking system can take braking maneuvers automatically if drivers fail to react [6]. Braking is the primary action of all these systems, but during brake, the torsos of the driver and occupant will lean forward due to the inertia force and become closer to the steering wheel and interiors, which may cause more severe injuries than those in the nominal seating position when the impact velocity remains the same. Even though the pretensioner has been invented to help restrain the occupant, the activation time of a traditional pyrotechnic pretensioner is after the impact and it is unlikely to restrain the occupant in the nominal seating position. What's more, considering the impact to the head of the driver when the airbag explodes, the injury severity may be tremendously increased. As a matter of fact, in the real driving scenarios, drivers are sometimes in a forward displaced position due to wrong driving postures, fatigue or the inertia force of braking. In this paper, sled tests are performed to verify the effects of a forward displaced position on the driver injuries in a frontal collision. Based on the test results, predictions about the possible injury or fatality risk in real traffic accidents are also made. 


\section{Sled Test}

The simulation of vehicle collisions in reality can be realized based on sled tests. Compared with real vehicle collision tests, sled test is a repeatable test approach with easy test preparations and operations. Different types of vehicles can be simulated through different lay-outs of interiors, steering wheels and seats, etc. The type of crash simulated is determined by the crash pulse generated by the waveform generator.

The sled lay-out is based on the interiors of an A-class passenger car. The whole sled includes the seat, the seat belt, the simulated steering wheel and the foot plate. An airbag with no exhaust vent is folded within the simulated steering wheel and it can be inflated to perform a frontal crash sled test with airbag. In the waveform generator, two kinds of polyurethane tubes are used to absorb the kinetic energy of the sled and thus decelerate it, which can simulate the two-stage acceleration curve of a typical frontal collision. The impact velocity of the sled is $40 \mathrm{kph}$ in the test. A hybrid III 50th percentile male dummy is placed on the seat. The injury responses during the crash are collected from the sensors installed on the dummy.

In this study, there are three seating positions for the dummy, namely the nominal seating position (Position 1), the $45 \mathrm{~mm}$ forward displaced seating position (Position 2) and the $85 \mathrm{~mm}$ forward displaced seating position (Position 3). Compared with the nominal seating position, in the forward displaced position only the dummy torso is rotated forward while the dummy pelvis is kept in its nominal position. Under different seating positions, tests with and without airbag are performed separately. A coat of sealant is applied to the inner airbag that has no exhaust vent, which makes it possible for the airbag to hold a constant air pressure. In the tests with airbag, the airbag is inflated before the crash and the air pressure is $0.1 \mathrm{MPa}$.

\section{Traffic Accident Statistics}

In the study, the selected traffic accident database is NASS CDS, which is managed by NHTSA. Data in NASS CDS are acquired from 24 monitoring sites throughout the US and only accidents with a certain severity are considered. About 5,000 accidents are investigated in detail each year and a weight is specified for every single accident. The weight is a reflection of how often a similar accident may happen in the US in a year.

In this paper, data collected from 1995-2007 are selected and filtered. This study focuses on both vehicles in frontal crash accidents and the striking vehicle in rear-end crash accidents. In the selected accidents, drivers should be properly belted and their injuries are observed and analyzed. The variable $\Delta \mathrm{V}$ reflects the velocity change before and after the collision and is one of the criteria for the crash severity. Among all the selected and analyzed accidents, $\Delta \mathrm{V}$ is between 38 to $42 \mathrm{kph}$ due to the fact that the impact velocity of the sled test is $40 \mathrm{kph}$. The drivers' avoidance maneuvers are also recorded in the database [7]. In this paper, it is assumed that the driver with an emergency braking maneuver ahead of collision will lean forward due to the inertia force, which is equivalent to the $85 \mathrm{~mm}$ forward displaced position in the sled tests. As for those drivers with no braking maneuvers, they are assumed to stay in the nominal seating position. In this paper, all the statistics results are weighted values.

\section{Injury responses and prediction}

The accelerations, forces and moments of different parts on the dummy in the process of collision are measured by the sensors. Injury responses of the dummy are then calculated based on these results. In the tests, the dummy head is likely to hit the simulated steering wheel and it will lead to a higher peak in the head acceleration curve as the steering wheel is apparently harder than the dummy head. In this study, a $15 \mathrm{~ms}$ time interval is used to calculated HIC, also known as $\mathrm{HIC}_{15}$ (15 ms head injury criterion). The value of HIC is determined by both the magnitude and the time duration of the head resultant acceleration, and its calculation formula is as follows ${ }^{[8]}$,

$$
H I C=\left[\frac{1}{t_{2}-t_{1}} \int_{t_{1}}^{t_{2}} a d t\right]^{2.5}\left(t_{2}-t_{1}\right)
$$


where $a$ is the resultant acceleration at the center of mass of the dummy head in g's, and $t_{1}$ and $t_{2}$ are the start and end time in milliseconds. The neck injury criterion Nij is a combination of neck axial tension/compression force and the neck flexion/extension moment. Nij is calculated as follows ${ }^{[9]}$,

$$
N_{i j}=\frac{F_{z}}{F_{\text {int }}}+\frac{M_{y}}{M_{\text {int }}}
$$

where $F_{\mathrm{z}}$ is the neck axial/compression force, $F_{\text {int }}$ is the intercept value of the axial force, $M_{\mathrm{y}}$ is the neck flexion/extension moment, and $M_{\text {int }}$ is the intercept value of the moment. The chest acceleration calculated in a $3 \mathrm{~ms}$ interval and the chest maximum deflection are employed to reflect the injury response of the dummy chest. Viano, etc studied the probabilities of getting injured on different body parts in traffic accidents and proposed the weighted injury criteria (WIC) ${ }^{[10]}$. WIC takes into consideration the injury values of head, chest, neck and femur, and the largest weight 0.53 is given to the head injury value in the WIC calculation formulas. In this study, the dummy head injury value is used as the principle criterion to estimate the probability of getting injured for the driver head in real accidents. The distribution relation between different $\mathrm{HIC}_{15}$ values and the injury severity levels in reality is obtained by a regression analysis of data acquired from cadaver tests performed by Prasad and Mertz ${ }^{[11]}$. The severity of injury is indicated by Abbreviated Injury Scale (AIS) and the estimation equations are as follows ${ }^{[12]}$,

$$
\left\{\begin{array}{l}
P(A I S 2+)=1 /\left(1+\exp ^{\left(2.49+140 / H I C-0.006 *^{*} H I C\right)}\right) \\
P(A I S 3+)=1 /\left(1+\exp ^{\left(3.39+140 / H I C-0.00531^{*} H I C\right)}\right) \\
P(A I S 4+)=1 /\left(1+\exp ^{\left(4.9+140 / H I C-0.00501^{*} H I C\right)}\right) \\
P(A I S 5+)=1 /\left(1+\exp ^{\left(7.82+140 / H I C-0.00613^{*} H I C\right)}\right)
\end{array}\right.
$$

\section{Results and discussion}

In total six sled tests are performed in this study. The data are collected and processed based on the SAE J211 Regulation. The six crash pulses have a good repeatability with a maximum acceleration of 20 g's and a duration of about $110 \mathrm{~ms}$.

The injury responses illustrated in Tables 1 4 are obtained by analyzing the data received from sensors on the dummy. In tests without airbag, the value of HIC15 is 204 for the dummy in the nominal seating position. The value of HIC15 increases as the dummy torso leans forward. HIC15 follows a similar change trend in tests with airbag, the value of which grows from 230 to 346 as the forward displaced distance increases. Chest acceleration does not show an obvious change with different forward displaced distances, but it can still be observed that the more forward the dummy is placed, the larger acceleration the dummy chest endures. Among the six sled tests, the dummy in its nominal seating position without airbag is compressed more than with airbag. One of the possible reasons is that the dummy doesn' thit the steering wheel during the whole collision process and the kinetic energy is mostly absorbed by the seat belt and thus the dummy chest is significantly compressed. While in other situations, the dummy head' $s$ impact with the steering wheel or airbag dissipates some energy, and as a result, the force exerted on the chest by the seat belt is relatively small. But compared with the other five test results, the chest deflection does increase as the dummy torso leans forward. Considering all the neck injury values, only the value of Nte in tests without airbag exceeds 0.5 while in other cases the same criterion is relatively low. As for the tests without airbag, the change rule of Nte is similar to that of other injury criteria above. Due to the fact that the airbag used in the tests has a different structure from the real airbag, a direct comparison of the results with or without airbag is not a wise choice. Thus, the results in the tests with airbag will be ignored in the following prediction of the distribution of different injury levels. 
Table $1 \mathrm{HIC}_{15}$ values in the tests

\begin{tabular}{|c|c|c|c|}
\hline HIC $_{15}$ & Position 1 & Position 2 & Position 3 \\
\hline W/out airbag & 204 & 226.6 & 235.22 \\
\hline With airbag & 230.44 & 287.9 & 345.57 \\
\hline
\end{tabular}

Table 2 Chest accelerations in the tests $(\mathrm{g})$

\begin{tabular}{|c|c|c|c|}
\hline Chest acce. & Position 1 & Position 2 & Position 3 \\
\hline W/out airbag & 30.7 & 30.83 & 32.68 \\
\hline With airbag & 29.44 & 31.44 & 31.19 \\
\hline
\end{tabular}

Table 3 Chest deflections in the tests ( $\mathrm{mm}$ )

\begin{tabular}{|c|c|c|c|}
\hline Chest deflection & Position 1 & Position 2 & Position 3 \\
\hline W/out airbag & 44.09 & 35.82 & 36.1 \\
\hline With airbag & 33.43 & 37.62 & 42.34 \\
\hline
\end{tabular}

Table 4 Nij values in the tests

\begin{tabular}{|c|c|c|c|c|}
\hline & Nij & Position 1 & Position 2 & Position 3 \\
\hline \multirow{4}{*}{ W/out airbag } & Ntf & 0.43 & 0.39 & 0.01 \\
\cline { 2 - 5 } & Nte & 0.29 & 0.48 & 0.75 \\
\cline { 2 - 5 } & Ncf & 0.32 & 0 & 0.3 \\
\cline { 2 - 5 } & Nce & 0.31 & 0 & 0.25 \\
\hline \multirow{4}{*}{ With airbag } & Ntf & 0.36 & 0.42 & 0.22 \\
\cline { 2 - 5 } & Nte & 0.27 & 0.4 & 0.33 \\
\cline { 2 - 5 } & Ncf & 0.34 & 0.31 & 0.34 \\
\cline { 2 - 5 } & Nce & 0.39 & 0.19 & 0.24 \\
\hline
\end{tabular}

The prediction of the driver head injury level using the HIC15 values obtained from the sled tests is discussed in the following paragraphs. According to Formula 3, the predicted distribution of the forward displaced driver head injury levels is represented by blue bars in Fig. 1.

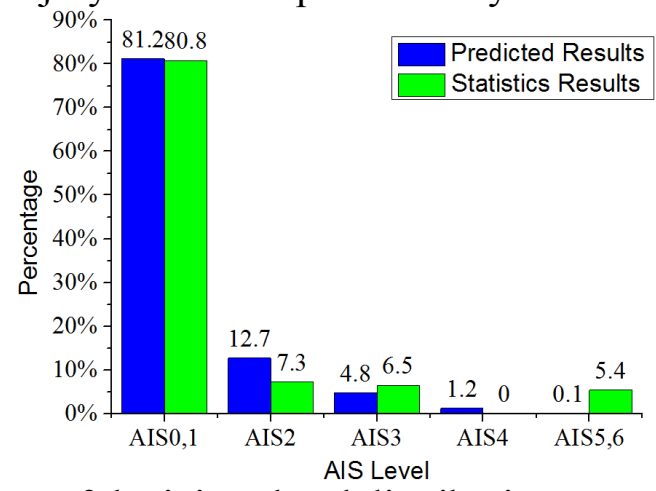

Fig. 1 Comparison of the injury level distribution - Prediction vs Reality

The vehicles involved in these accidents have either an unignited airbag or no airbag at all. According to the NASS CDS database, among accidents from 1995 to 2007 with a $\Delta \mathrm{V}$ between 38 to $42 \mathrm{kph}$, the distribution of properly belted adult driver head injury levels under forward displaced conditions is illustrated in Fig. 1 by green bars. Airbags of the vehicles involved have the same conditions as just described. By comparison, the predicted distribution is close to the statistical distribution in real accidents when AIS is no more than 4. If AIS is greater than 4, there is a large difference between the two distributions. The main reason is that, on one hand, the number of samples in the cadaver test is not sufficient for the regression analysis in getting Formula 3. On the other hand, 
the number of samples is far too few. Under the filtering conditions in this paper, there is only one case left. In the recorded accidents with drivers in the nominal seating positon and vehicles meeting the same filtering requirements above, the number of samples is also too far from enough to do any statistical analysis.

\section{Conclusions}

Drivers can become forward displaced when driving due to wrong driving postures, fatigue or the inertia force caused by braking. Through sled tests, the statement that the forward displaced position caused by the inertia force during emergency braking will result in a more severe injury on the body regions of head, neck and chest is verified. Using the injury responses of the dummy head from the tests, the distribution of head injury levels in real accidents is predicted. With this method and computer simulations, a fast and accurate overall estimate of the injury distribution can be performed and this is very helpful in offering a more comprehensive assessment of occupant protection effects of vehicle safety technologies. This research technique can also provide a basis for the control strategy establishment of vehicle safety devices.

\section{Acknowledgements}

This work was supported by the National Natural Science Foundation of China (Grant no. 51305223), and Science and Technology Project of Suzhou (Grant no. SYG201426).

\section{References}

[1] J.H. Zhang, H.L. Du, C.S. Ma, Automotive Design Crash Safety, Beijing, 2010.

[2] F. Muehlfeld, I. Doric, R. Ertlmerer, Statistical behavior modeling for driver-adaptive precrash systems, IEEE Transactions on Intelligent Transportation Systems. 14(4) (2013) 1764-1772.

[3] T. Kim, H. Jeong, Crash probability and error rates for head-on collisions based on stochastic analyses, IEEE Transactions on Intelligent Transportation Systems. 11(4) (2010) 896-904.

[4] K. Kusano, H. Gabler, Safety benefits of forward collision warning, brake assist, and autonomous braking systems in rear-end collisions, IEEE Transactions on Intelligent Transportation Systems. 13(4) (2012) 1546-1555.

[5] A. Balint, H. Fagerlind, A. Kullgren, A test-based method for the assessment of pre-crash warning and braking systems, Accident Analysis and Prevention. 59 (2013) 192-199.

[6] E. Bertolazzi, F. Biral, L. Da L, Supporting drivers in keeping safe speed and safe distance: the SASPENCE subproject within the European framework programme 6 integrating project PReVENT, IEEE Transactions on Intelligent Transportation Systems. 11(3) (2010) 525-538.

[7] X. Yan, R. Harb, E. Radwan, Anglyses of factors of crash avoidance maneuvers using the general estimates system, Traffic Injury Prevention. 9(2) (2010) 173-180.

[8] J. Versace, A Review of the Severity Index, 710881. Detroit: SAE, 1971.

[9] S. Massoud, B. Janet, S. Varun, Effect of seat belts equipped with pretensioners on rear seat adult occupants in high-severity rear impact, 2008-01-1488. Detroit: SAE, 2008.

[10]D. Viano, S. Arepally, Assessing the safety performance of occupant restraint systems, 902328. Detroit: SAE, 1990.

[11]P. Prasad, H. Mertz, The position of the United States delegation to the ISO working group 6 on the use of HIC in the automotive environment, 851246. Detroit: SAE, 1985. 
[12]R. Eppinger, E. Sun, F. Bandak, Development of improved injury criteria for the assessment of advanced automotive restraint system II. Washington DC: NTHSA, 1999. 